\title{
Remarques sur l'identification et la preuve d'une pratique ultérieure établissant un accord des Etats ou des organisations internationales sur l'interprétation d'un traité
}

Fecha de recepción: 9 de enero de 2016

Fecha de aceptación: 5 de agosto de 2016

Doi: 10.12804/revistas.urosario.edu.co/acdi/a.5295

\section{Maurice Kamto*}

Résumé: Cette contribution porte sur l'analyse des articles 31 et 32 de la Convention de Vienne du 23 mai 1969 sur le droit des traités (CVT) relativement aux moyens d'interprétation. Selon l'auteur, l'article 31 \$3 prévoit deux types d'accords, respectivement à ses alinéas a) et b). Celui qui intéresse la présente réflexion est l'accord résultant d'une pratique ultérieure, au sens de l'article $31 \$ 3, \mathrm{~b})$. La distinction entre au titre de l'article $31 \$ 3$, b) et celui au titre de l'article 32 ne semble pas avoir été bien perçue par la Commission du droit international (CDI) dans ses travaux sur le sujet, «Les accords et la pratique ultérieurs dans le contexte de l'interprétation des traités», comme le montre le projet de Conclusion 6 dont le paragraphe1. Les critères d'identification de l'existence d'une pratique ultérieure établissant un accord sur l'interprétation d'un traité se dégagent de la jurisprudence, la preuve de l'acceptation d'une pratique ultérieure comme établissant un

* Professeur à l'Université de Yaoundé II (Cameroun) Membre et ancien Président de la Commission du droit international Membre de l'Institut de droit international. Email: mkamtots@yahoo.fr

Para citar este artículo: Kamto, M., "Remarques sur l'identification et la preuve d'une pratique ultérieure établissant un accord des Etats ou des organisations internationales sur l'interprétation d'un traité", Anuario Colombiano de Derecho Internacional (ACDI), 2017, 10, pp. 25-49. Doi: 10.12804/revistas.urosario.edu.co/acdi/a.5295 
accord sur l'interprétation d'un traité aussi. Si celle-ci est stricte en ce qui concerne la pratique des Etats, on note plutôt une certaine souplesse dans l'établissement d'un accord sur l'interprétation d'un traité résultant de la pratique ultérieure des organisations internationales. Aux termes de son analyse, l'auteur conclut que la conception trop lâche de la notion d'accord, retenue par le Rapporteur spécial - et suivie pour l'instant par la CDI - qui considère qu'un «accord» en droit international ne conduit pas nécessairement à un engagement contraignant, ne lui paraît pas convaincante.

Mots clés: accord, interprétation, traités, pratique ultérieure, pratique ultérieure des Etats et des organisations internationales, identification, preuve.

Comentarios sobre la identificación y la prueba de una práctica posterior que establece un acuerdo entre Estados

o entre organizaciones internacionales relativo a la interpretación de un tratado

Resumen: esta contribución hace un análisis sobre el derecho a los tratados relativo a los medios de interpretación de los artículos 31 y 32 de la Comisión de Viena del 23 de mayo de 1969. Para el autor, el artículo 31, numeral 3, prevé dos tipos de acuerdos relacionados con los literales a) y b). En lo específico, el de mayor interés para esta reflexión es el acuerdo que resulta de una práctica posterior del artículo 31, numeral 3, literal b). La distinción entre el título del artículo 31, numeral 3, literal b) y el artículo 32 no parece haber sido bien analizada por la Comisión de Derecho Internacional (CDI) en sus trabajos sobre el tema Los acuerdos y la práctica posterior o ulterior en el contexto de la interpretación de tratados, como se muestra en la conclusión 6 del párrafo 1. Los criterios de identificación de la existencia de una práctica posterior establecen que un acuerdo sobre la interpretación de un tratado se aparta de la jurisprudencia. La prueba de la aceptación de una práctica ulterior establece, igualmente, un acuerdo sobre la interpretación de un tratado. Si esta es estricta en lo que concierne a la práctica de los Estados, se nota más bien cierta flexibilidad en el establecimiento de un acuerdo relativo a la interpretación de un tratado, que resulta de la práctica posterior de las organizaciones internacionales. En los términos de su análisis, el autor concluye que la concepción muy amplia del acuerdo retenida por el relator especial que ha sido seguida por 
la CDI y que considera que 'un acuerdo' en derecho internacional no conduce necesariamente a una obligación vinculante no le parece convincente.

Palabras clave: acuerdo, interpretación, tratados, práctica posterior o práctica ulterior, práctica ulterior de Estados y de organizaciones internacionales, identificación, prueba.

Comments on the Identification of and Evidence Supporting Subsequent Practice Establishing an Agreement of States or International Organizations as to the Interpretation of a Treaty

Abstract: This contribution focuses on the analysis of Articles 31 and 32 of the 23 May 1969 Vienna Convention on the Law of Treaties (VCLT) in relation to means of interpretation. According to the author, article 31 s 3 provides for two types of agreements, respectively under subparagraphs a) and b). This reflection concerns itself with the agreement resulting from subsequent practice within the meaning of Article 31\$3, b). The distinction between what comes under Article 31 ( 3, b) and that under Article 32 does not appear to have been well received by the International Law Commission (ILC) in its work on the subject titled "Agreements and Subsequent Practice in the Context of Treaty Interpretation", set out in Conclusion 6 Paragraph 1. The criteria for identifying the existence of a subsequent practice so as to establish an agreement on the interpretation of a treaty emerge from the case law. Evidence of acceptance of a subsequent practice also establishes an agreement on the interpretation of a treaty. If there is strictness regarding State practice, there is more flexibility in the establishment of an agreement on the interpretation of a treaty resulting from the subsequent practice of international organizations. According to his analysis, the author concludes that the loose interpretation of the concept of agreement adopted by the Special Rapporteur - and followed so far by the Commission - which considers that "agreement" in international law does not necessarily lead to a binding commitment, is not convincing.

Key words: Agreement, interpretation, treaties, subsequent practice, subsequent practice of states and international organizations, identification, evidence. 
Comentários sobre a identificação e a prova de uma prática posterior que estabelece um acordo entre Estados ou entre Organizações Internacionais, relativa à interpretação de um tratado

Resumo: Esta contribuição faz uma análise sobre o direito aos tratados relativo aos meios de interpretação dos artigos 31 e 32 da Comissão de Viena do 23 de maio de 1969. Para o autor, o artigo 31, numeral 3 prevê dois tipos de acordos relacionados com os literais a e b. No específico, o de maior interesse para esta reflexão é o acordo que resulta de uma prática posterior do artigo 31, numeral 3 literal b. A distinção entre o título do artigo 31, numeral 3 literal b e o artigo 32 não parece ter sido bem analisada pela Comissão de Direito Internacional (CDI) nos seus trabalhos sobre o tema Os acordos e a prática posterior ou ulterior no contexto da interpretação de tratados, como se mostra na conclusão 6 do parágrafo 1. Os critérios de identificação da existência de uma prática posterior estabelecem que um acordo sobre a interpretação de um tratado aparta-se da jurisprudência. A prova da aceitação de uma prática ulterior estabelece, igualmente, um acordo sobre a interpretação de um tratado. Se esta é estrita no que concerne à prática dos Estados, nota-se sobretudo certa flexibilidade no estabelecimento de um acordo relativo à interpretação de um tratado, que resulta da prática posterior das organizações internacionais. Nos termos da sua análise, o autor conclui que a concepção muito ampla do acordo retida pelo relator especial que tem sido seguida pela CDI e que considera que "um acordo" em direito internacional não conduz necessariamente a uma obrigação vinculante, não lhe parece convincente.

Palavras-chave: Acordo, interpretação, tratados, prática posterior ou prática ulterior, prática ulterior de Estados e de organizações internacionais, identificação, prova.

\section{Introduction}

On dit de l'interprétation en droit tantôt qu'elle est un art, tantôt qu'elle est une science. Elle est peut-être un peu tout cela à la fois. Elle est assurément une technique qui permet de rechercher le sens et la portée d'un terme ou d'un énoncé juridique. Elle n'est pas laissée à l'arbitraire de l'interprète. Elle obéit à certaines règles dont la quintessence a été consignée dans les 
articles 31 et 32 de la Convention de Vienne du 23 mai 1969 sur le droit des traités (CVT). Comme on le sait, alors que l'article 31 pose la règle générale d'interprétation, dont la Cour internationale de Justice (CIJ) a dit qu'elle consacrait le droit international coutumier, ${ }^{1}$ l'article 32 pour sa part détermine les moyens complémentaires d’interprétation.

Ces deux articles énumèrent plusieurs moyens d'interprétation, mais, à notre avis, pas exactement les mêmes moyens, sinon il n'y aurait aucun intérêt à y consacrer deux articles distincts. L'article 31 \$3 prévoit deux types d'accords, respectivement à ses alinéas a) et b). L'accord dont il est question à l'alinéa a) est un accord qui, bien qu'il ne soit pas nécessaire qu'il satisfasse à toutes les exigences de forme prévues par la CVT, exige pour sa formation l'accomplissement d'un minimum de formalités qui lui confèrent l'autorité juridique nécessaire. ${ }^{2}$ L'accord qui intéresse la présente réflexion est celui résultant d'une pratique ultérieure, au sens de l'article 31§3, b) qui dispose: "Il sera tenu compte, en même temps que du contexte: (...); b) De toute pratique ultérieurement suivie dans l'application du traité par laquelle est établi l'accord des Parties à l'égard de l'interprétation du traité».

L'exhortation à faire «appel à des moyens complémentaires d'interprétation» contenue dans l'article 32 est formulée en des termes tellement larges qu'il est permis de penser que lesdits moyens peuvent

\footnotetext{
1 Application de la Convention pour la prévention et la répression du crime de génocide (Bosnie-Herzégovine c. Serbie-Monténégro) CIJ, Recueil 2007, p. 43, \$160; Différend relatif à des droits de navigation et des droits connexes (Costa Rica c. Nicaragua) CIJ Recueil $2009,213, \S 47$.

2 Dans la sentence rendue le 13 juillet 2014 dans l'affaire de la Délimitation maritime entre l'Inde et le Bangladesh, le Tribunal arbitral constitué sous l'égide de la CPA a déclaré dans ce sens: «\$165: The Tribunal is not convinced that the clear determination of the Bagge Award was undone by the exchange of correspondence between officials of the two governments in 1951. As noted by Bangladesh, the Indian letter was unsigned. While recognizing that a subsequent agreement in the sense of article 31(3)(a) of the Vienna Convention on the Law of Treaties need not itself possess all the formalities of a treaty (see International Law Commission, Report on the Sixty-Fifth Session, UN Doc. A/68/10 at p. 32 (2013), the Tribunal does not consider the exchange of letters to be sufficiently authoritative to constitute such a subsequent agreement between the Parties. Above all, it is difficult for the present Tribunal to accept that such a low-level and brief exchange of correspondence between civil servants, purporting to reverse an important general determination of the formal Indo-Pakistani Boundary Disputes Tribunal established by a solemn agreement at the Inter-Dominion Conference at New Delhi on 14 December 1948, represents an authentic agreement of the Parties»—Sentence en ligne sur le site de la Cour permanente d'arbitrage (CPA) -
} 
inclure également des accords. Mais, à notre avis, il ne peut s'agir du même type d'accords que ceux qui sont visés à l'article 31 a) et b), sinon il y a aurait une redondance injustifiée en ce sens qu'un moyen d'interprétation ne saurait relever à la fois de la «règle générale» et des «moyens complémentaires». Ainsi, peut-on considérer comme faisant partie de ces moyens complémentaires d'interprétation visés à l'article 32 , « toute autre pratique ultérieure» (autre que celle prévue à l'article $31 \$ 3$ b), entendue comme «toute conduite d'une ou plusieurs personnes parties à l'application du traité, après la conclusion de celui-ci».

Cette distinction ne semble pas avoir été bien perçue par la Commission du droit international (CDI) dans ses travaux sur le sujet "Les accords et la pratique ultérieurs dans le contexte de l'interprétation des traités» comme le montre le projet de Conclusion 6 dont le paragraphe1 énonce que "(l)'identification des accords ultérieurs et de la pratique ultérieure au sens de l'article 31, paragraphe 3, exige, en particulier, de déterminer si les parties, par un accord ou une pratique, ont pris position au sujet de l'interprétation d'un traité». ${ }^{3}$

Cette formulation donne à croire qu'en soi, une pratique ultérieure, au sens de l'article $31 \leqq 3 \mathrm{~b}$ ), peut constituer un moyen autonome d'interprétation. Or, il n'en est rien: une telle pratique n'est pertinente aux fins d'interprétation d'un traité que pour autant qu'elle établit l' «accord des Parties à l'égard de l'interprétation du traité». Autrement dit, la pratique ultérieure au sens de la disposition précitée de la CVT est la preuve d'un accord, mais aussi sa manifestation; cet accord ne revêt pas la forme de celui visé à l'article $31 \$ 3$ a) qui, lui, n’est pas nécessairement fondé sur une pratique ou ne résulte pas de celle-ci. Faute d'opérer cette distinction, la CDI n'a pas estimé nécessaire de formuler un projet de conclusion sur la preuve d'une pratique ultérieure établissant l'accord des Parties à l'égard de l'interprétation d'un traité. La jurisprudence internationale fournit pourtant de la matière à ce sujet.

Il convient de distinguer à cet égard entre l'accord résultant de la pratique des Etats de celui découlant de la pratique des organisations internationales. La pratique de ces dernières est envisagée ici par rapport à l'interprétation de leurs actes constitutifs. En principe, les règles prévues aux articles $31 \$ 3$ et 32 sont également applicables dans le cas de l'interprétation

Rapport de la Commission du droit international sur les travaux de sa Soixante-dixième session (A/70/10). Documents officiels. Soixante-dixième session. Supplément nº10, p. 94. 
des actes constitutifs des organisations internationales. La question qui se pose dans ce cas est de savoir comment on établit l'accord résultant de la pratique d'une organisation. L'examen de cette question atteste de l'existence d'une différence entre les modes d'expression de l'accord dans la pratique des Etats et celle des organisations. Dans le cas des Etats, il ressort de la jurisprudence internationale une exigence de la preuve d'une acceptation expresse d'un accord résultant de la pratique ultérieure entre les Etats Parties à un traité (I). Dans le cas des organisations internationales, on note une certaine souplesse en la matière (II) tenant essentiellement au mode de prise de décision au sein de ces entités juridiques.

\section{Les critères d'identification d'une pratique ultérieure établissant un accord entre Etats Parties sur l'interprétation d'un traité}

La question de l'identification de la pratique ultérieure a été abordée par la CDI en des termes par trop généraux qui éludent une question de fond: celle de la preuve non seulement de l'existence d'une telle pratique, mais de ce qu'elle établit un accord entre les parties au sujet de l'application ou de l'interprétation du traité. Il échet donc avant tout de poser clairement les termes du problème que soulève cette question (A). On s'apercevra alors que plus que le simple fait d'avoir "pris position», en l'occurrence par une pratique ultérieure, au sujet de l'interprétation d'un traité, cette pratique, pour être pertinente à cette fin, doit remplir certains critères (B) dont la preuve n'est pas toujours aisée, mais peut être établie selon diverses modalités (C).

\section{A. Position du problème}

L'identification d'un accord ultérieur ou d'une pratique ultérieure est abordée dans le projet de Conclusion 6 de la CDI adopté en 2014 comme si elle obéissait aux mêmes critères dans un cas comme dans l'autre. Le commentaire de ce projet de Conclusion confirme cette observation et établit clairement que la Commission, suivant en cela l'approche choisie par le Rapporteur spécial sur le sujet, ne fait pas de distinction catégorielle entre l'accord ultérieur au sens du paragraphe 3, a) de l'article 31 et la pratique ultérieure au sens du paragraphe 3, b) du même article. On peut y lire ce qui suit: «Pour déterminer qu'un accord ultérieur ou une pratique ultérieure au sens des alinéas $a$ et $b$ du paragraphe 3 de l'article 31 
représente une prise de position quant à l'interprétation d'un traité, il est souvent nécessaire de procéder à une soigneuse analyse factuelle et juridique $» .^{4}$ Cette pétition sur la démarche à suivre n'est pas incorrecte sauf qu'elle ne saurait justifier l'approche indiscriminée adoptée aussi bien dans le projet de Conclusion lui-même que dans le commentaire.

Or, la distinction entre accord ultérieur et pratique ultérieure n'est ni fortuite, ni à fonction esthétique. Elle renvoie à deux procédés distincts de formation d'un accord au sens du droit international. Dans le cas de l'accord ultérieur, la question de l'identification renvoie à la question de savoir si un accord est bien ce qu'il prétend être, c'est-à-dire si un acte est né de la rencontre entre la volonté des deux ou de plusieurs Etats, en l'occurrence au sujet de l'interprétation d'un traité, ou si l'intention des Parties de former un tel acte peut être déduite des circonstances. Dans l'affaire de l'île Kasikili/Sedudu (Botswana/Namibie) par exemple, le Botswana soutenait qu'une décision prise, en décembre 1984, de réaliser une étude conjointe, et l'ensemble des instruments en relation avec cette décision, constituaient un «accord intergouvernemental» intervenu entre les Parties au sujet de l'application du traité de 1890 relatif entre autres à la frontière entre les deux Etats. La Cour, après examen des documents en question, «est parvenue à la conclusion qu'il n'y a pas eu, entre l'Afrique du Sud et le Botswana, d'accord 'au sujet de ... l'application (du traité) de $1890 » .^{5}$

Dans le cas de la pratique ultérieure, il s'agit de savoir si un comportement d'un ou de plusieurs Etats a conduit à un accord de ces Etats au sujet de l'interprétation du traité ayant inspiré le comportement en question.

Si dans certaines circonstances il n'est pas toujours aisé d'identifier un accord, ultérieur ou non, entre les Etats, il paraît encore plus difficile de rapporter la preuve d'un accord résultant d'une pratique ultérieure. La jurisprudence internationale, en particulier celle de la CIJ, a apporté à ce sujet des clarifications qui auraient mérité l'attention de la CDI dans son travail sur «Les accords et la pratique ultérieurs dans le contexte de l'interprétation des traités». La position de la Cour à ce sujet a été exprimée dans un dictum de son arrêt rendu le 13 décembre 1999 en l'affaire de l'̂̂le Kasikili/Sedudu.

4 Rapport de la Commission du droit international sur les travaux de sa Soixante-dixième session (A/69/10). Documents officiels. Soixante-neuvième session. Supplément n¹0, p. 178.

5 Arrêt du 13 décembre 1999, CIJ, Recueil 1999, p. 1091, \69. 
Dans cette affaire, les Parties avaient invoqué à l'appui de leurs thèses respectives la conduite ultérieure des Parties au Traité de 1890. Pour la Namibie, cette conduite ultérieure consistait dans «le contrôle et l'utilisation de l'île de Kasikili par les Masubia de la bande de Caprivi, l'exercice de la juridiction sur l'île par les autorités responsables namibiennes et le silence gardé par le Botswana et ses prédécesseurs pendant près d'un siècle en pleine connaissance de cause... ». ${ }^{6} \mathrm{La}$ Cour s'est attachée à «rechercher si la présence très ancienne, et qui n'a pas soulevé d'objection, de membres de la tribu des Masubia» sur l'île litigieuse «est constitutive d'une pratique ultérieurement suivie dans l'application du traité (de 1890) par laquelle est établi l'accord des parties à l'égard de l'interprétation du traité» (CVT, 31\$3, b). ${ }^{7}$ Elle pose à cet effet les balises suivantes: « Pour qu'une telle pratique puisse être démontrée, il faudrait au moins que deux éléments soient établis: d'une part, que l'occupation de l'île par les Masubia ait participé de la conviction des autorités du Caprivi que la frontière fixée par le traité de 1890 suivait le chenal sud du Chobe et, d'autre part, que ce fait ait été pleinement connu et accepté par les autorités du Bechuanaland comme confirmant la frontière fixée par le traité». ${ }^{8}$

Bien que formulés dans un cas d'espèces et pas en terme généraux, il est permis d'y voir les deux critères formant le principe directeur de la preuve en la matière. Après tout, la Cour ne s'exprime presque jamais abstraitement, de façon académique, mais toujours en rapport avec le différend qui lui est soumis. Il n'empêche que l'on dégage de ses décisions des principes et autres critères juridiques d'origine jurisprudentielle. S'il est relativement aisé d'identifier une pratique, il est en revanche souvent plus difficile de prouver l'acceptation par les parties d'une pratique comme constituant une interprétation ou une application d'un traité, au sens des dispositions précitées de la CVT. Il échet dès lors d'examiner, d'abord les éléments indissociables selon lesquels la pratique doit être connue et acceptée par les autorités de l'Etat, avant d'examiner l'épineuse question de la preuve de l'acceptation.

\footnotetext{
$6 \quad$ Ibid., p. 1093, \ 72.

7 Ibid., p. 1093, \ 73 .

$8 \quad$ Ibid., p. 1093, $\int 74$.
} 


\section{B. Critères de l'existence d'une pratique ultérieure établissant un accord sur l'interprétation d'un traité}

Deux critères sont indispensables à l'identification d'une pratique ultérieure comme établissant un accord sur l'interprétation d'un traité: d'une part, la pratique doit être connue, officiellement pour ainsi dire, par les autorités des Etats Parties; d'autre part, cette pratique doit être acceptée par lesdites autorités étatiques. Bien qu'il soit intellectuellement possible de dissocier ces deux éléments, la Cour les associe et, de fait, ils sont étroitement liés l'un à l'autre. On les examinera donc ensemble.

D’emblée, précisons que pour qu'un comportement ou un acte soit constitutif d'une pratique pertinente au sens de l'article 31\$3, b) CVT, il faut qu'il soit attribuable à l'Etat, suivant le mécanisme d'attribution prévu dans les Articles sur la responsabilité de l'Etat pour fait internationalement illicite. ${ }^{9}$ Pour que la pratique identifiée soit pertinente aux fins de l'établissement d'un accord sur l'interprétation d'un traité, l'on doit pouvoir apporter la preuve de sa connaissance et de son acceptation par les autorités étatiques. Ces deux éléments ou critères que sont la connaissance et l'acception sont inséparables en ce que l'on ne peut accepter que ce que l'on connaît.

L'arrêt rendu par la CIJ en 1999 en l'affaire de l'Île Kasikili/Sedudu est particulièrement éclairant au sujet de ces deux éléments. Dans cette affaire, chacune des deux Parties avait invoqué des faits ou des évènements qui, selon elle, constituaient une pratique ultérieure établissant un accord sur l'interprétation du traité de 1890 délimitant la frontière entre elles. Dans son mémoire, le Botswana avait invoqué en ce sens le rapport Eason, suscitant la contestation de la Namibie qui fit notamment valoir que la Grande-Bretagne n'avait formulé aucune revendication sur la base de ce rapport, alors que ses échanges de correspondance avec l'Allemagne au sujet du reste de la frontière méridionale s'étaient poursuivis jusqu’à l'éclatement de la première guerre mondiale. La Cour a suivi la position de

\footnotetext{
9 V. les Articles de la CDI sur la responsabilité de l'Etat pour fait internationalement illicite (2001), Partie I, Chapitre II; le projet de Conclusion 5 (L'attribution d'une pratique ultérieure) sur «Les accords et la pratique ultérieurs dans le contexte de l'interprétation des traités». Rapport de la Commission du droit international sur les travaux de sa Soixante-dixième session (A/70/10) op. cit., p. 94; voir aussi le deuxième rapport sur L'identification du droit international coutumier, par Wood, M., Rapporteur spécial, doc. A/CN.4/672, 22 mai 2014, p. 16, \34.
} 
la Namibie sur ce point, estimant que «le rapport Eason et les circonstances qui l'entourent ne sauraient être considérés comme constitutifs d'une «pratique ultérieurement suivie dans l'application du traité» de 1890, au sens de l'alinéa b) du paragraphe 3 de l'article 31 de la convention de Vienne. Elle relève en effet que ledit rapport semble n'avoir jamais été communiqué à l'Allemagne et avoir toujours conservé un caractère interne». De surcroît, le gouvernement britannique lui-même n'avait jamais donné une suite à ce rapport, «ni après son établissement [...], ni ultérieurement». ${ }^{10}$

Pour sa part, la Namibie invoquait des cartes de la région figurant la frontière autour de l'île de Kaiskili/Sedudu dans le chenal sud du Chobe. La Cour a relevé que ces cartes n'interprétaient pas officiellement le traité de 1890 et que si la ligne frontière qui y avait été figurée dans le chenal sud, «c'était compte tenu de la présence intermittente sur l'île de populations venues de la bande de Caprivi». De l'avis de la Cour «rien ne montre que cette présence ait eu un lien avec les prétentions territoriales capriviennes», le mouvement transfrontière des populations n'étant pas un phénomène inhabituel en Afrique. Certes, dans leur rapport du 19 janvier 1948, les administrateurs Trollope et Redman, prenaient acte de ce que l'île litigieuse était «'utilisée par les membres des tribus de la parties orientale de la bande de Caprivi' sans objection du Bechuanaland »; de même, un rapport d'étude conjointe sur le Chobe, établi le 15 juillet 1985 par les experts de l'Afrique du Sud et du Botswana, dans le cadre des discussions sur le cours de la frontière autour de l'île querellée relevait que le bétail du Caprivi traversait le fleuve à la nage lorsque le pâturage faisait défaut du côté de Caprivi, indiquant au passage que «les séjours sur l'île étaient devenus plus rares au cours des dernières années ». ${ }^{11}$ Toutefois, non seulement le capitaine Eason de la police du Bechuanaland affirmait dès 1912 que le chenal nord devait être considéré comme le chenal principal du Chobe autour de l'île Kasikili/Sedudu, plaçant de la sorte l'île en territoire du Bechuanaland au regard du traité de 1890, mais il semble, comme le dit la Cour, que du côté du Bechuanaland, puis du Botswana, «la présence intermittente des Masubia sur l'île n'ait jamais inquiété personne et ait été tolérée, à tout le moins, parce qu'elle n'apparaissait pas liée à une interprétation des termes du traité de $1890 .{ }^{12}$ Autrement dit, une certaine pratique ultérieure sur la

\footnotetext{
10 CIJ Rec. 1999, pp. 1077-1078, \55.

11 Ibid., p. 1094, \ 74.

12 Ibid., p. 1095, \ 74.
} 
frontière et au sujet de l'utilisation de l'île a existé et était connue des autorités du Bechuanaland puis du Botswana. Mais, elle n'était manifestement pas acceptée comme une interprétation du traité de 1890. Elle n'établissait donc pas un accord des Parties au sujet de l'interprétation dudit traité et n'est dès lors pas pertinente à cette fin. Aussi, la Cour a-t-elle conclu sur ce point que «l'utilisation paisible ouverte, pendant des années, de l'île de Kasikili/Sedudu par les membres de la tribu des Masubia du Caprivi oriental n'est pas constitutive d'une "pratique ultérieurement suivie dans l'application du traité» de 1890, au sens de l'alinéa b) du paragraphe 3 de l'article 31 de la convention de Vienne sur le droit des traités». ${ }^{13}$

La Cour a également considéré comme ne pouvant «être regardés comme constitutifs » de ladite pratique, d'autres faits et incidents additionnels invoqués par les Parties, qu'il s'agisse des textes instituant la réserve animalière qu'elle considère comme des textes constituant des «documents internes» qui, de surcroît, «ne font pas expressément référence à l'île Kasikili/Sedudu»; de l'allégation selon laquelle le chef de l'Etat botswanais de l'époque se serait rendu sur l'île en 1972, et dont le Botswana reconnaît lui-même qu'elle n'est pas établie; ou enfin, de l'insuffisance de la preuve de l'incident mentionnée par la Namibie, consistant en l'appréhension, à la même époque, par les gardes botswanais, de trois ou quatre Capriviens dans l'île sous le chef de braconnage, et qui furent relâchés par un magistrat du Botswana, après une détention de cinq jours, «au motif qu'ils avaient été arrêtés sur un territoire qui ne relevait pas de la juridiction du Botswana». ${ }^{14}$

En somme, la Cour en arrive à la conclusion que «la conduite ultérieure des parties au traité de 1890 n'a donné lieu à aucun «accord ... entre les parties au sujet de l'interprétation du traité ou de l'application de ses dispositions », au sens de l'alinéa a) du paragraphe 3 de l'article 31 de la convention de Vienne de 1969 sur le droit des traités, et qu'elle n'a pas davantage donné lieu à une quelconque "pratique ... suivie dans l'application du traité par laquelle est établi l'accord des parties à l'égard de l'interprétation du traité», au sens de l'alinéa b) de cette même disposition».. ${ }^{15}$

\footnotetext{
13 Ibid., p. 1095 , $\ 75$.

14 Ibid., pp. 1095-1096, $\mathbb{S} 77$ et 78.

15 Ibid., p. 1096, \ 79 .
} 


\section{Preuve de l'acceptation d'une pratique ultérieure comme établissant un accord sur l'interprétation d'un traité}

L'acceptation peut être expresse ou tacite, une acceptation tacite pouvant prendre la forme de l'acquiescement ou du silence.

L'acceptation expresse ne soulève pas de problèmes particuliers. Elle se fait sous diverses formes d'expression formelle du consentement, le droit international n'exigeant pas en la matière des formes particulières, sauf lorsqu'un instrument juridique exige l'accomplissement d'une ratification, laquelle se fait suivant les procédures propres à chaque Etat.

Il en va différemment de l'acceptation tacite. Celle-ci est généralement déduite des circonstances qui entourent une pratique ultérieure, en particulier du comportement de l'Etat vis-à-vis d'une telle pratique. On distinguera à cet égard l'hypothèse de l'acquiescement, qui est admise comme un mode d'expression du consentement en droit international, du silence dont le statut juridique est controversé.

En ce qui concerne l'acquiescement, la jurisprudence constante de la CIJ et des instances arbitrales internationales anciennes et récentes admettent qu'un Etat peut exprimer son consentement à un acte par un comportement qui, au vu des circonstances, permet d'établir qu'il n'est pas opposé à cet acte ou qu'il le regarde comme s'imposant à lui. Ainsi, dans l'affaire du Temple de Préah Vihéar (Cambodge c. Thaïlande), alors que la Thailande, bien qu'ayant reçu des cartes que les autorités françaises lui avaient communiquées, arguait de ce que cette communication était «pour ainsi dire unilatérale», et qu'elle n'avait pas été «invitée à en accuser formellement réception» et dès lors ne l'avait pas fait, la Cour a déclaré: «En réalité [...], un accusé de réception très net ressort incontestablement de la conduite de la Thailande; mais s'il n'en avait pas été ainsi, il est clair que les circonstances étaient de nature à appeler dans un délai raisonnable une réaction de la part des autorités siamoises, au cas où celles-ci auraient voulu contester la carte ou auraient eu de graves questions à soulever à son égard. Or, elles n'ont réagi ni à l'époque ni pendant de nombreuses années et l'on doit, de ce fait, conclure à leur acquiescement. Qui taoet consentire videtur si loqui debuisset ac potuisset». ${ }^{16}$

Toutefois, l'acquiescement est toujours une affaire d'espèce et des circonstances qui l'entourent, notamment la conduite des Etats. Elle ne

16 Arrêt du 15 juin 1962, CIJ, Rec. 1962, p. 23. 
saurait donner lieu à une théorie générale abstraction faite des circonstances concrètes; c'est un exemple typique où seule l'appréciation in concreto d'un acte ou d'une situation permet de procéder à une qualification juridique. Concrètement, il apparaît que l'acquiescement ne peut être retenu dans certaines hypothèses telles que celle de la modification tacite d'une frontière déjà délimitée ou l'acquisition de la souveraineté sur un territoire relevant d'un souverain disposant d'un titre territorial formel. Ainsi, dans l'affaire de la Frontière terrestre et maritime entre le Cameroun et le Nigeria, aux prétentions du Nigeria fondées sur la théorie de «la consolidation historique du titre» par l'occupation des parties litigieuses du territoire camerounais, notamment dans la zone du lac Tchad et dans la péninsule de Bakassi, et l'acquiescement du Cameroun à cette occupation, la Cour note que «dès lors qu'elle a conclu que la frontière dans le lac Tchad se trouvait délimitée bien avant que ne débutent les travaux de la CBLT (Commission du Bassin du lac Tchad), les éventuelles effectivités nigérianes doivent être considérées, du point de vue de leurs conséquences juridiques, comme des actes contra legem»». ${ }^{17} \mathrm{La}$ Cour ajoute plus loin, au sujet des effectivités invoquées par le Nigeria pour justifier ses revendications territoriales dans la zone du lac Tchad: «Certaines de ces activités - organisations des services publics de santé et d'enseignement, maintien de l'ordre, administration de la justice - pourraient normalement être considérés comme des actes accomplis à titre de souverain. [...] Cependant [...] puisque le Cameroun détenait un titre préexistant sur cette région du lac, le critère juridique applicable est l'existence ou non d'un acquiescement manifeste du Cameroun au transfert de son titre au Nigeria». ${ }^{18}$ Sur ce point, la Cour a rappelé le fameux dictum de sa jurisprudence de principe dans l'affaire du Différend frontalier (Burkina Faso/Mali), ${ }^{19}$ dont le passage pertinent à cet égard est le suivant: «Dans le cas où le fait ne correspond pas au droit, où le territoire objet du différend est administré effectivement par un Etat autre que celui qui possède le titre, il y a lieu de préférer le titulaire du titre ${ }^{20}$ Autrement dit, on ne saurait voir dans la non-protestation d'un titulaire du titre de souveraineté sur un territoire ou d'un titre juridique

\footnotetext{
17 Arrêt du 10 octobre 2002, CIJ, Rec. 2002, p. 350, \$ 64.

18 Ibid., p. 352, \6 67.

19 CIJ Rec. 1986, p. 587, \63.

20 Ibid., p. 352, \68; voir aussi Différend territorial (Lybie/Tchad), CII Rec. 1994, p. 38, $\int 75-76$.
} 
sur la frontière, aux effectivités d'un autre Etat, un acquiescement à une modification de facto dudit titre; ni une pratique ultérieurement suivie dans l'application du traité délimitant la frontière, par laquelle est établi un accord des Parties à l'égard de l'interprétation dudit traité, au sens de l'alinéa b) du paragraphe 3 de l'article 31 CVT. Toute non-réaction à un comportement susceptible d'appeler une réaction ne vaut donc pas acquiescement à ce comportement, quand bien même celui-ci serait une pratique au sens du droit international. ${ }^{21}$

S’agissant précisément du silence, les opinions divergent encore sur ses possibles effets juridiques en droit international. On vient de voir que, dans les cas des activités en rapport avec les titres frontaliers et territoriaux, le silence ne prête pas à conséquence juridique, quand bien même les activités en question «pourraient normalement être considérés comme des actes accomplis à titre de souverain $»{ }^{22} \mathrm{Il}$ ne s'agit cependant que d'un cas spécifique non généralisable. On ne peut en effet répudier de façon générale le silence comme mode d'expression de la volonté et plus précisément du consentement des Etats. A la lumière de la pratique, il apparaît que le silence peut exprimer le refus ou l'acquiescement et qu'il joue un rôle non négligeable dans la formation, l'interprétation et la modification des rapports internationaux. ${ }^{23}$ Le silence est envisagé ici non pas comme une pratique négative ou une abstention, mais comme une inaction ou une absence de réaction face à un comportement ou une pratique qui appelle une réaction. En ce sens, on l'a qualifié de «réaction inactive (ou passive)» en ce qu'il serait au sein des comportements passifs ou inactifs, «celui qui fait suite, et est envisagé comme une réaction à une sollicitation extérieure». ${ }^{24}$

La connaissance de la pratique ultérieure appelant une réaction est une condition pour que l'absence de réaction de l'Etat dont la réaction est sollicitée par cette pratique produise l'effet juridique de l'acceptation.

21 Sur la pratique en ce sens, voir notamment: SFDI, La pratique et le droit international, colloque de Genève, A. Pedone, 2004.

22 Sur l'opinion que l'absence de réaction, ou le silence, dans ce cas ne vaut pas acquiescement, voir notamment Bedjaoui, M., opinion dissidente dans l'affaire de la Délimitation de la frontière maritime entre la Guinée-Bissau et le Sénégal, Sentence du 31 juillet 1989, RSA, vol. XX, part II, p. 181 \70.

$23 \mathrm{~V}$. à ce sujet la thèse de doctorat, très complète et bien articulée de Marie, A., Le silence de l'Etat comme manifestation de sa volonté, Université de Panthéon-Assas (Paris 2), 2013.

24 Ibid., p. 19, \ 16. 
Elle constitue dans ce cas le critère d'identification de la volonté de l'Etat. Peu importe les modalités de la connaissance; il suffit qu'elle soit prouvée pour que le silence déploie pleinement ses effets juridiques. ${ }^{25}$ Ainsi, comme l'a souligné l'Organe d'appel de l'OMC dans l'affaire des Poulets désossés, il n'est pas exclu "que, dans des situations spécifiques, l' «absence de réaction» ou le silence d'une partie particulière à un traité puisse, compte tenu des circonstances entourant l'affaire, être comprise comme une acceptation de la pratique suivie par d'autres Parties. De telles situations peuvent se présenter lorsqu'une partie qui n'a pas suivi une pratique a eu connaissance ou a été informée de la pratique suivie par d'autres Parties (par exemple, au moyen d'une notification ou à l'occasion de sa participation à une réunion où cette pratique a été examinée, mais ne réagit pas $\gg{ }^{26}$

En plus du critère fondamental de la connaissance d'un comportement, en l'occurrence de la pratique alléguée, il y a lieu de se demander si, toujours au vu des circonstances d'une espèce, l'Etat dont on veut attacher des effets juridiques à son silence était en mesure de réagir. Cet élément n'est pas négligeable, dans un contexte international où l'on a affaire, d'une part, à certains Etats dont la faiblesse des ressources et des compétences limite considérablement leur participation efficiente à la gestion des rapports internationaux, d'autre part, de plus en plus à des Etats déstabilisés qu'une certaine terminologie nomme des «Etats défaillants», qui sont dans l'incapacité parfois durable d'assurer une telle participation.

Quoi qu'il en soit, le haut niveau de certitude qu'exige l'établissement d'un accord ou du consentement d'un Etat à une pratique contraste avec un certain degré de souplesse observé dans l'établissement d'un accord des Etats membres d'une organisation internationale sur une pratique ultérieure de celle-ci relativement à l'application ou à l'interprétation de son acte constitutif.

25 V. notamment affaire de la Souveraineté sur Pedra Branca (Singapour c. Malaisie), CIJ Rec. 2008, \274. La Cour relève que: «La Malaisie et ses prédécesseurs n’ont jamais réagi à ce comportement ni à d'autres formes de comportement de même nature [...] et qui toutes (sauf en ce qui concerne l'installation matérielle de communication) avaient été portées à sa connaissance».

26 Affaire des Poulets désossés (CE - Classification donanière des morceaux de poulets désossés et congelés), Rapport de l'Organe d'appel du 12 septembre 2005, \ 272. 


\section{Une certaine souplesse dans l'établissement d'un accord sur l'interprétation d'un traité résultant de la pratique ultérieure des organisations internationales}

L'applicabilité de l'article 31, paragraphe $3 \mathrm{~b}$ ), aux actes constitutifs d'organisations internationales découle de l'article 5 de la CVT qui stipule que cette Convention s'applique aux actes constitutifs des organisations internationales. La CIJ en a fait application dans diverses affaires. Dans son avis consultatif relatif à la Licéité de l'utilisation des armes nucléaires par un État dans un conflit armé notamment, après avoir qualifié les actes constitutifs d'organisations internationales de «traités d'un type particulier», la Cour a indiqué, en guise d'introduction à son interprétation de la Constitution de l'OMs, que «conformément à la règle coutumière d'interprétation qui a trouvé son expression à l'article 31 de la convention de Vienne de 1969 sur le droit des traités, les termes des traités doivent être interprétés «dans leur contexte et à la lumière de [1']objet et $\mathrm{d}[\mathrm{u}]$ but [du traité]», et il doit être «tenu compte, en même temps que du contexte: [...]; b) [d]e toute pratique ultérieurement suivie dans l'application du traité par laquelle est établi l'accord des Parties à l'égard de l'interprétation du traité ». ${ }^{27}$ Après avoir cité différents précédents tirés de sa propre jurisprudence, dans lesquels elle avait notamment eu recours comme moyen d'interprétation à la pratique ultérieure au sens de l'article 31, paragraphe 3 b), elle a déclaré qu'elle «en fera[it] également application en la présente espèce aux fins de déterminer si, d'après la Constitution de l'oms, la question à laquelle il lui a[vait] été demandé de répondre se pos[ait] «dans le cadre de [l']activité» de cette organisation $»{ }^{28}$

La CDI s'est appuyée sur la disposition précitée de la CVT ainsi que sur la pratique jurisprudentielle pour énoncer dans le paragraphe $1 \mathrm{du}$ projet de Conclusion 11 de son projet sur les Accords et la pratique ultérieure dans le contexte de l'interprétation des traités que «(l)es articles 31 et 32 s'appliquent à un traité qui est l'acte constitutif d'une organisation internationale». Elle ajoute au paragraphe 3 du même projet de Conclusion que la pratique ultérieure d'une organisation internationale dans l'application de son acte

\footnotetext{
27 Ibid.

28 Ibid.
} 
constitutif peut contribuer à l'interprétation de cet acte, lors de l'application des deux articles précités de la CVT. ${ }^{29}$

Deux questions se posent alors: d'une part, à quelles conditions les actes d'une organisation internationale peuvent-ils être considérés comme une pratique ultérieure de l'organisation à cette fin? D'autre part, quels types d'actes d'une organisation internationale peuvent-ils exprimer sa pratique ultérieure au sens de l'article 31 \$3, b) de la CVT ? C'est à ces deux questions que s'efforcera de répondre la présente partie, en examinant successivement les conditions pour que les actes d'une organisation internationale constituent la pratique ultérieure de cette organisation au sens des dispositions précitées de la CVT (A) et les types d'actes des organisations internationales et leur aptitude à exprimer la pratique ultérieure d'une organisation (B).

\section{A. Les conditions pour que des actes d'une organisation internationale constituent sa pratique ultérieure au sens de 1'article $31 \$ 3, b)$ de la CVT}

La pratique d'une organisation internationale peut traduire un accord sur l'application ou l'interprétation de son acte constitutif. Cette pratique se manifeste à travers les actes de ses organes. Théoriquement, il y a lieu de penser qu'il peut s'agir des actes de l'organe plénier; mais on ne peut exclure l'idée que ceux de l'organe exécutif de l'organisation, ou plus largement ceux de ses organes principaux puissent jouer un tel rôle. Ainsi, s'agissant de l'ONU par exemple, il n'est pas illogique de penser que les résolutions de l'Assemblée générale, mais aussi celles du Conseil de sécurité puissent constituer la pratique de cette organisation. Le débat reste en revanche ouvert en ce qui concerne les actes du Secrétaire général des Nations Unies.

Les actes susceptibles d'exprimer la pratique d'une organisation doivent, d'une part, s'inscrire dans le cadre des activités de cette organisation, d'autre part, ne pas être isolés. C'est ce qui ressort notamment de l'avis consultatif sur la Licéité de l'utilisation des armes nucléaires par un Etat dans un conflit armé. (OUI, il faut enlever la CIJ) En effet, en ce qui concerne l'objet de la pratique ou le lien indispensable entre celle-ci et le domaine d'activité de l'organisation, la Cour, après avoir examiné la pratique de l'oms, déclare

29 Rapport de la Commission du droit international sur les travaux de sa Soixante-dixième session (A/70/10), op. cit., p. 96. 
qu' "(a)ucun des rapports et résolutions visés dans le préambule de la résolution WHA46.40 de l'Assemblée mondiale de la Santé ne participent d'une pratique de l'oms dans le domaine de la licéité de l'utilisation des armes nucléaires ». ${ }^{30}$ Selon elle, «semblable pratique ne saurait être déduite, en particulier, de passages de certaines résolutions de l'Assemblée mondiale de la Santé évoquées au cours de la $[\ldots]$ procédure $[. .]. »{ }^{31}$ Pour la Cour, il n'y a rien de surprenant à ce que l'oms, en tant que sujet de droit international, «soit amenéeà en appliquer les règles de droit international ou s'intéresse à leur développement; mais il ne s'ensuit pas qu'elle ait reçu mandat, au-delà des termes de sa constitution, pour traiter elle-même de la licéité ou de l'illicéité de l'utilisation de l'armement nucléaire ». ${ }^{32}$

S’agissant du caractère isolé de l'acte évoqué au titre de la pratique, la Cour affirme: «La résolution WHA46.40 elle-même, adoptée, non sans opposition, dès que la question de la licéité de l'utilisation des armes nucléaires a été soulevée au sein de l'OMs, ne saurait être considérée comme exprimant ou constituant à elle seule une pratique qui établirait un accord entre les États membres de l'Organisation pour interpréter sa constitution comme l'habilitant à traiter de la question de la licéité de l'utilisation des armes nucléaires ». 33

\section{B. Les types d'actes des organisations internationales et leur aptitude à exprimer la pratique ultérieure d'une organisation}

De façon générale, on classe les actes des organisations internationales en deux grandes catégories: les actes contraignants et les actes non contraignants. Cette summa divisio ne rend pas toujours compte de toute la variété desdits actes. Entre les deux grandes catégories, il existe une zone grise où certains actes en principe, ou formellement, non contraignants acquièrent une force contraignante.

En ce qui concerne les actes contraignants, il semble plus logique de les considérer comme exprimant un accord ultérieur plutôt qu'une pratique ultérieure. Telle est du reste la tendance qui se dessine en pratique. Ainsi dans l'avis consultatif OMs du 8 juillet 1996 précité, en se demandant si une

\footnotetext{
30 Avis consultatif du 8 juillet 1996, CIJ Rec. 1996, p. 81, 』 27.

31 Ibid.

32 Ibid. p. 82, \ 27.

33 Ibid. $\mathrm{N}^{\text {os }}$ italiques.
} 
résolution donnée d'un organe exprimait ou constituait «une pratique qui établirait un accord entre les États membres de l'Organisation", la Cour, citant l'article 31, paragraphe 3 b), a choisi de faire primer l'accord des parties au traité sur la pratique de l'organe en question. ${ }^{34}$

La question est plus délicate s'agissant des actes non contraignants d'une organisation internationale. D'abord, il est permis de se demander si un acte juridiquement non contraignant d'une telle organisation peut constituer une pratique ultérieure. L'examen de la pratique permet de répondre par l'affirmative à cette question. Dans son avis consultatif sur les Conséquences juridiques de l'édification d'un mur dans le territoire palestinien occupéla CIJ a montré l'évolution de l'interprétation de l'article 12 de la Charte des Nations Unies à travers les résolutions de l'Assemblée générale dont on sait qu'elles sont de simples recommandations non contraignantes pour les Etats membres, sauf lorsque l'Assemblée, constatant la défaillance du Conseil de sécurité à s'acquitter de sa responsabilité principale en matière de maintien de la paix et de la sécurité internationales adopte en cette matière une résolution s'imposant à tous les Etats membres, à l'instar de la résolution 377 A (V). En effet, si pendant un certain temps l'Assemblée générale s'était refusée de recommander certaines mesures concernant le maintien de la paix et de la sécurité internationales dès lors que le Conseil de sécurité était saisi de la situation, cette interprétation de l'article 12 «devait cependant évoluer par la suite». ${ }^{35} \mathrm{De}$ fait, la Cour a relevé «l'existence d'une tendance croissante à voir l'Assemblée générale et le Conseil de sécurité examiner parallèlement une même question relative au maintien de la paix et de la sécurité internationales (voir, par exemple, les cas de Chypre, de l'Afrique du Sud, de l'Angola, de la Rhodésie du Sud et, plus particulièrement de la Bosnie-Herzégovine et de la Somalie) $»{ }^{36}$ La Cour estime dès lors que "la pratique acceptée de l'Assemblée générale telle qu'elle a évolué, est compatible avec le paragraphe 1 de l'article $1^{\text {er }}$ de la

34 La Cour permanente de Justice internationale avait déjà adopté cette approche dans l'affaire relative à la Compétence de l'Organisation internationale du travail pour réglementer accessoirement le travail personnel du patron, avis consultatif, CPII Rec. Série B n 13, p. 19 et 20; voir Engel, S., " "Living" International Constitutions and the World Court (the Subsequent Practice of International Organs under their Constituent Instruments)», International and Comparative Law Quarterly, vol. 16 (1967), p. 871.

35 Avis consultatif du 9 juillet 2004, CIJ Rec. 2004, p. 149, \$ 27.

36 Ibid., p. 149-150, \27. 
Charte $» .{ }^{37}$ En l'occurrence, l'acceptation de la pratique découle sans doute des conditions de l'adoption des différentes résolutions de l'Assemblée générale établissant sa pratique ultérieure relativement à l'interprétation de l'article 12, mais aussi de la réponse du conseiller juridique de l'ONU à une question posée par le Pérou lors de la $23^{\mathrm{e}}$ session de l'Assemblée, qui confirma que cette dernière «avait interprété les mots «remplit ... les fonctions » employés à l'article 12 de la Charte comme signifiant «remplit ... les fonctions en ce moment». ${ }^{38}$ Ainsi, la position donnée au nom des Etats membres quant à la pratique suivie par l'Organisation permet-elle d'établir l'accord au sujet de l'interprétation d'une disposition donnée de son traité constitutif. ${ }^{39}$

Dans le cas de cet avis consultatif sur le Mur, les résolutions constitutives de la pratique portaient sur une matière relavant du Chapitre VII de la Charte sous l'empire duquel les résolutions ont une force contraignante. On pourrait être enclin à penser qu'un accord sur une pratique ultérieure fondée sur de telles résolutions est relativement facile à établir en raison du caractère même de ces actes. Si un tel raisonnement n'est pas sans intérêt, il apparaît à la lumière de la pratique des organisations internationales que l'accord sur une pratique ultérieure peut également résulter du mode et/ ou des conditions d'adoption des actes non contraignants en question. Il en est ainsi en particulier lorsque des actes «recommandatoires» ont été adoptés par consensus ou à l'unanimité. Ainsi, dans l'affaire relative à la Chasse à la baleine dans l'Antarctique la CIJ, après avoir relevé que l'Australie et la Nouvelle-Zélande surestimaient l'importance juridique des résolutions et lignes directrices de la Commission baleinière qu'elles invoquaient a, certes, déclaré que «(c)es instruments ne sauraient être considérés comme constitutifs d'un accord ultérieur au sujet de l'interprétation de l'article VIII, ni d'une pratique ultérieure établissant un accord des parties à l'égard de l'interprétation du traité au sens des alinéas $a$ ) et $b$ ), respectivement, du paragraphe 3 de l'article 31 de la Convention de Vienne sur le droit des traités ». Mais, faisant référence aux recommandations, non contraignantes,

\footnotetext{
37 Ibid., p. $150, \int 28$.

38 Ibid, p. 149, \ 27.

39 V. commentaire du projet de Conclusion $11 \mathrm{du}$ projet de conclusions sur «Les accords et la pratique ultérieurs dans le contexte de l'interprétation des traités » Rapport de la Commission du droit international sur les travaux de sa Soixante-dixième session (A/70/10), op. cit., p. 105.
} 
de ladite Commission, la Cour a précisé que lorsqu'elles sont «adoptées par consensus ou à l'unanimité, elles peuvent être pertinentes aux fins de l'interprétation de la convention ou du règlement qui lui est annexé».40 En l'occurrence, le consensus ou l'unanimité expriment l'opinio juris des Etats membres, laquelle confère à un acte formellement non contraignant une force juridique qui le rend opposable à tous les Etats membres et, ce faisant, établit soit l'existence d'un accord au sens de l'alinéa a) du paragraphe 3 de l'article 31 susvisé, soit plus sûrement un accord sur la pratique ultérieure au sens de son alinéa $b$ ).

Est-ce à dire qu'une série de résolutions, par nature non contraignantes, adoptées sur une certaine période même à une large majorité ne pourrait pas constituer une pratique ultérieure pertinente ? La question ne manque pas d'intérêt dans la mesure où certains auteurs estiment que la répétition des résolutions de cette nature peut être à la base d'une opinio juris pouvant conduire à la formation d'une règle coutumière. Mais, comme on l'a vu, l'article $31 \$ 3$, a) et b) vise dans ces deux alinéas, respectivement un accord, et une pratique ultérieure par laquelle est établi un accord. Or, il est admis que la règle de droit international coutumier n'est pas établie sur la base de l'accord entre les Etats qui participent au processus coutumier. Il s'ensuit que l'inexistence d'un accord entre ces Etats sur une pratique ultérieure ne permet pas de considérer cette pratique comme pertinente aux fins de l'interprétation d'un traité. L'accord ultérieur, qu'il s'agisse d'un acte formel ou acte accompli avec un minimum de formalité nécessaire à l'établissement de son autorité juridique, ou qu'il résulte d'une pratique ultérieure ne saurait être présumé, même lorsqu'il est fondé sur les actes des organisations internationales. Il doit pouvoir être prouvé, sinon un Etat pourrait s'opposer à une interprétation d'un traité auquel il est partie, qui serait fondé sur un accord ne reflétant pas son consentement ou son acquiescement, quelle que soit la forme que pourrait prendre celui-ci. Comme on l'a vu, il pourrait revêtir dans certains cas la forme du silence, pourvu qu'il soit possible de rapporter la preuve d'un acquiescement dans ce silence. Dans ces conditions où le consentement de l'Etat est indispensable à la formation de l'accord ultérieur tant au sens de l'alinéa a) du paragraphe de l'article 31 CVT, qu'au sens de son alinéa b), il parait bien

40 Arrêt du 31 mars 2014, \46; voir Deuxième rapport sur les accords et la pratique subséquente dans le contexte de l'interprétation des traités, par M. Georg Nolte, Rapporteur spécial, A/CN./671, 26 mars 2014, spéc. \55 et s. 
difficile de suivre la CDI - qui elle-même a suivi sur ce point le Rapporteur spécial sur les «Accords et la pratique ultérieurs dans le contexte de l'interprétation des traités » - lorsqu'elle admet l'idée qu'un accord ultérieur au sens de l'article 31 peut être non contraignant non seulement pour les parties, mais aussi pour l'interprète. ${ }^{41}$

Il paraît bien difficile, au moins d'un point de vue pratique, pour une juridiction de se considérer non liée par un accord par lequel toutes les parties à un traité auraient retenu une certaine interprétation du traité. Cela vaut également d'ailleurs dans les situations où l'accord ultérieur intervient en dehors des procédures institutionnelles prévues pour l'interprétation du traité. A preuve, dans l'affaire Etats-Unis - Mesures affectant la production et la vente des cigarettes aux clous de girofle dans laquelle se posait la question de l'effet interprétatif à accorder à la Déclaration ministérielle de Doha, l'organe d'appel a estimé, dans un premier temps, que cette Déclaration ne constituait pas une «interprétation multilatérale» au sens de l'article IX:2 de l'accord OMC, qui prévoit que la conférence ministérielle et le Conseil général de l'OMC peuvent adopter des interprétations contraignantes. ${ }^{42}$ Puis, dans un second temps, l'organe d'appel a considéré que la Déclaration de Doha pouvait tout à fait constituer un accord ultérieur au sens de l'article 31 (3) de la CV de 1969, en se fondant sur le projet de la CDI de 1966 sur le droit des traités. Selon ce projet d'articles, tout accord qui «influe spécifiquement» sur l'interprétation d'un traité, et qui par ailleurs «exprime clairement une communauté de vues et une acceptation de cette communauté de vues», «doit», selon l'organe d'appel, être pris en compte dans l'interprétation. Tel est en effet le critère qui a été retenu par l'organe d'appel, lequel a insisté sur ce dernier point en citant de nouveau le projet de la CDI de 1966 qui avait indiqué qu'un tel accord ultérieur «doit être réputé incorporé au traité aux fins de son interprétation». Or, si de la sorte, l'accord ultérieur ou résultant de la pratique ultérieure oblige l'interprète, il serait bien curieux qu'il ne soit pas contraignant pour les Etats parties au traité eux-mêmes.

La conception trop lâche de la notion d'accord, retenue par le Rapporteur spécial - et suivie pour l'instant par la CDI - qui considère qu'un

\footnotetext{
41 V. dans le même sens, l'intervention de Forteau, M., sur le Deuxième rapport sur «les Accords et la pratique ultérieurs dans le contexte de l'interprétation des traités» par M. Georg Nolte, Rapporteur spécial, 2015.

42 V. OMC, ORD décision du 4 avril 2012 (WT/DS406/AR/R), V. sur ce point les observations de Forteau, M., dans son intervention précitée.
} 
«accord» en droit international ne conduit pas nécessairement à un engagement contraignant, ne me parait dès lors pas convaincante. A vrai dire, il me semble même franchement critiquable, en particulier dans le contexte de l'article $31 \mathrm{CVT}$. Aussi, faut-il espérer qu'une telle conception ne prospère pas au-delà de la première lecture du projet de conclusions de la Commission sur les «Accords et la pratique ultérieurs dans le contexte de l'interprétation des traités», en tout cas qu'elle ne soit pas retenue dans la mouture finale qui sera adoptée en seconde lecture.

\section{Références}

\section{Ouvrages}

Marie, A., Le silence de l'Etat comme manifestation de sa volonté, Université de Panthéon-Assas (Paris 2), 2013.

Nolte, G. (Ed.) Treaties and Subsequent Practice, Oxford (Oxford University Press) 2013 (393 pp.).

\section{Revues et Publications périodiques}

Engel, S., "Living" International Constitutions and the World Court (the Subsequent Practice of International Organs under their Constituent Instruments), International and Comparative Law Quarterly, 1967, 16, (4), p. 865-910.

Sur la pratique en ce sens, voir notamment: SFDI, La pratique et le droit international, Colloque de Genève, Paris, Pedone, 2004.

\section{Jurisprudence}

\section{Cour Internationale de Justice}

\section{Avis Consultatifs:}

Conséquences juridiques de 1'édzjîcation d'un mur dans le territoire palestinien occupé, avis consultatif; CIJ Recueil 2004.

Licéité de la menace ou de l'emploi d'armes nucléaires, avis consultatif, CIJ Recueil 1996. 
Affaire du temple de Préah Vihéar (Cambodge v. Thailande), Fond, .Arrêt du 15 juin 1962: CIJ Recueil 1962.

Application de la convention pour la prévention et la répression du crime de génocide (Bosnie-Herzégovine v. Serbie-et-Monténégro), arrêt, CIJ Recueil 2007.

Chasse à la baleine dans l'Antarctique (Australie v. Japon; Nouvelle-Zélande (intervenant)), arrêt, CIJ Recueil 2014.

Différend frontalier, arrêt, CIJ Recueil 1986.

Différend relatif à des droits de navigation et des droits connexes (Costa Rica v. Nicaragua), arrêt, CIJ Recueil 2009.

Frontiére terrestre et maritime entre le Cameroun et le Nigéria (Cameroun v. Nigéria; Guinée équatoriale (intervenant)), arrêt, CIJ Recueil 2002. Ile de Kasikili/Sedudu (Botswana/Namibie), arrêt, CIJ Recueil 1999.

\section{Arbitrages}

Affaire des Poulets désossés (CE - Classification douanière des morceaux de poulets désossés et congelés), Rapport de l'Organe d'appel du 12 septembre 2005.

Permanent Court of Arbitration The Hague, Bay of Bengal Maritime Boundary Arbitration between Bangladesh and India, 7 July 2014.

\section{Organisation Mondiale de Commerce}

\section{Documents}

Commission du droit international, A/CN./671, 26 mars 2014, spéc. par. 55 et s.

Rapport de la Commission du droit international sur les travaux de sa Soixante-dixième session (A/70/10). Documents officiels. Soixantedixième session. Supllémnet $\mathrm{n}^{\circ} 10$.

Rapport de la Commission du droit international sur les travaux de sa Soixante-dixième session (A/69/10). Documents officiels. Soixanteneuvième session. Supllémnet $\mathrm{n}^{\circ} 10$.

V. OMC, ORD décision du 4 avril 2012 (WT/DS406/AR/R), V. sur ce point les observation de M. Forteau dans son intervention précitée. 
